\title{
EFFICIENT AND ROBUST APPROACH FOR THE DERIVATION OF CLOSED-FORM GREEN'S FUNCTIONS
}

\author{
M.I. Aksun \\ Bilkent University \\ Dept. of Electrical \& Electronics Eng.
}

Ankara 06533 TURKEY

\begin{abstract}
Spatial domain Green's functions for multilayer, planar geometries are cast into closed forms with two-level approximation of the spectral domain representation of the Green's functions. This approach is very robust and much faster compared to the original one-level approximation.
\end{abstract}

\section{Introduction}

In the application of the spatial domain MoM to the solution of mixed-potential integral equation (MPIE), one needs to calculate the Green's functions of the vector and scalar potentials in the spatial domain where they are represented as oscillatory integrals, called Sommerfeld integrals. The evaluation of these integrals are quite time consuming, therefore the matrix-fill time would be significantly improved if these integrals can be evaluated efficiently. Recently, a technique was proposed to approximate these integrals analytically for a horizontal electric dipole over a thick substrate backed by a ground plane, and called closed-form Green's functions [1] This technique was improved first for two layer geometries with arbitrary thicknesses [2], then for multilayer geometries with sources of HED, HMD, VED and VMD [3]. However, a question remains to be answered on the robustness and the efficiency of the technique, because some of the Green's functions are usually difficult to approximate and it is recommended that the function to be approximated needs to be examined in advance. The difficulties in this technique are originated by the approximation of the spectral domain Green's functions in terms of complex exponentials. Because of these difficulties, the technique of getting the closed-form Green's functions and subsequently using them in the MoM applications are considered to be not robust and could not be used much for the development of a general purpose electromagnetic software. In this paper, a new approach based on a twolevel approximation is proposed to overcome these difficulties, and demonstrated that it is very robust and computationally efficient.

II. two-level approach for approximating the spectral domain Green's function

To alleviate the necessity of investigating the spectral domain Green's functions in advance and the difficulties caused by the trade-off between the sampling range $T_{\circ}$

Supported in part by NATO's Scientific Affairs Division in the framework of the Science for Stability Programme 
and the sampling period, the approximation is performed in two levels. The first part of the approximation is performed along the path $C_{a p 1}$ while the second part is done along the path $C_{a p 2}$, as shown in Fig. 1, where the parametric equations describing the paths $C_{a p 1}$ and $C_{a p 2}$ for the first and second parts of the approximation, respectively are given by

$$
\begin{array}{ll}
\text { For } C_{a p 1} & k_{z_{i}}=-j k_{i}\left[T_{\circ 2}+t\right] \quad 0 \leq t \leq T_{\circ 1} \\
\text { For } C_{a p 2} & k_{z_{i}}=k_{i}\left[-j t+\left(1-\frac{t}{T_{\mathrm{o} 2}}\right)\right] \quad 0 \leq t \leq T_{\circ 2}
\end{array}
$$

where $t$ is the running variable and sampled uniformly on the corresponding range. The use of the two-level approach can be explained by giving the following procedure:

- Choose $T_{\mathrm{o} 2}$ such that $k_{\rho_{\max 2}} \geq k_{m}$ where $k_{m}$ is the maximum value of the wavenumber involved in the geometry: For example, assume that $\mathrm{GaAs}$ is a material with a highest dielectric constant $\left(\epsilon_{r}(G a A s)=12.5\right)$ among possible substrate materials, then $k_{m}=\sqrt{12.5} k_{\circ}$, and $T_{\mathrm{o} 2}=5$ is chosen.

- Choose $T_{\circ 1}$, i.e., $k_{\rho_{\max 1}}=k\left[1+\left(T_{\circ 1}+T_{\circ 2}\right)^{2}\right]^{1 / 2}$, and the number of samples on $\left[k_{\rho_{\max 2}}, k_{\left.\rho_{\text {max }}\right]}\right]$ : The choices of $T_{01}$ is not very critical as long as one chooses $k_{\rho_{\max }}$ large enough to pick up the behavior of the spectral domain Green's function for large $k_{\rho}$ and since the spectral domain behaviors of the Green's functions are always smooth beyond $k_{\rho_{\max 2}}$ it is not necessary to have a large number of samples on $\left[k_{\rho_{\max 2}}, k_{\rho_{\max 1}}\right] . T_{01}=400$ and the number of samples is chosen to be 50 .

- Sample $\tilde{G} / j 2 k_{z_{i}}$ along the path $C_{a p 1}$ and apply the GPOF method [4]:

$$
\begin{aligned}
f\left(k_{z_{i}}\right)\left(\cong \frac{\tilde{G}}{j 2 k_{z_{i}}} \text { for } k_{\rho} \in\left[k_{\rho_{\max 2}}, k_{\rho_{\max 1}}\right]\right) & =\sum_{n=1}^{N_{1}} b_{1 n} e^{\beta_{1 n} t} \\
& =\sum_{n=1}^{N_{1}} a_{1 n} e^{-\alpha_{1 n} k_{z_{i}}} \\
\alpha_{1 n}=\frac{\beta_{1 n}}{j k_{i}} ; \quad a_{1 n}=b_{1 n} e^{-j k_{i} \alpha_{1 n} T_{02}} &
\end{aligned}
$$

where $b_{1 n}$ and $\beta_{1 n}$ are the coefficients and the exponents obtained from the GPOF method, and $N_{1}$ is the number of exponentials used in this approximation. The choice of the number of exponentials is based upon the number of significant singular values obtained in an intermediate step of the application of the GPOF method. For this specific problem, five exporientials are chosen to approximate the Green's function on the range of $k_{\rho} \in\left[k_{\rho_{\max 2}}, k_{\rho_{\max 1}}\right]$.

- Subtract the approximating function $f\left(k_{z_{i}}\right)$ from the original function $\tilde{G} / j 2 k_{z_{i}}$ : The remaining function will be non-zero over a small range of $k_{\rho}\left(\in\left[0, k_{r h o_{\text {max }}}\right]\right)$ so that one can pick up the fine features of this funtion without employing a huge number of sampling points.

- Sample the remaining function along the path $C_{a p 2}$ : Since the maximum range for the sampling $\left(k_{\rho_{\max 2}}\right)$ is rather small, compared to that of the one-level 
approximation scheme, the frequency of sampling can be made quite high without substantially increasing the number of samples. For all practical purposes (including the worst case situation) the choice of 200 as the number of samples would be more than enough to get a good approximation.

$$
\begin{gathered}
\frac{\tilde{G}}{j 2 k_{z_{i}}}-f\left(k_{z_{i}}\right) \cong \sum_{n=1}^{N_{2}} b_{2 n} e^{\beta_{2 n} t}=\sum_{n=1}^{N_{2}} a_{2 n} e^{-\alpha_{2 n} k_{z_{i}}} \\
\alpha_{2 n}=\frac{\beta_{2 n} T_{02}}{k_{i}\left(1+j T_{02}\right)} ; \quad a_{2 n}=b_{2 n} e^{k_{i} \alpha_{2 n}}
\end{gathered}
$$

where $b_{2 n}$ and $\beta_{2 n}$ are the coefficients and exponents of the exponentials of $t$ obtained from the application of the GPOF method, and $a_{2 n}$ and $\alpha_{2 n}$ are the coefficients and the exponents of the exponentials of $k_{z_{i}}$. The number of exponentials $N_{2}$ in this part of the approximation is chosen again by the number of significant singular values.

The two approximation techniques are applied to $\tilde{G}_{z x}^{A} / j k_{x}$ for a geometry of four layers at $30 \mathrm{GHz}$ (1st layer- PEC; 2nd layer- $\epsilon_{r 2}=12.5, d_{2}=0.03 \mathrm{~cm}$; 3rd layer$\epsilon_{r 3}=2.1, d_{3}=0.07 \mathrm{~cm}$; 4th layer- free-space, and the source and observation planes are chosen at the interface of second and third layers) and compared for the CPU time on SPARCstation 10/41, using the same number of total exponentials (=13) for different approximation parameters, and presented in a table format below:

$\begin{array}{ccc}\text { Approximation } & \text { Approximation Parameters } & \text { CPU time (sec) } \\ \text { one-level } & T_{\mathrm{o}}=200, N_{s}=400 & 198.0 \\ \text { one-level } & T_{\mathrm{o}}=200, N_{s}=500 & 382.0 \\ \text { two-level } & T_{\mathrm{o} 1}=400, N_{s_{1}}=50 T_{\mathrm{o} 2}=5, N_{s_{2}}=50 & 1.2 \\ \text { two-level } & T_{\mathrm{o} 1}=400, N_{s_{1}}=50 T_{\mathrm{o} 2}=5, N_{s_{2}}=100 & 3.5\end{array}$

where $N_{s}$ is the number of samples in one-level approximation scheme while $N_{s 1}$ and $N_{s 2}$ are the number of samples of the first and second parts of the approximation, respectively, in two-level approximation approach. it is obvious that the two-level approximation approach improves the computational efficiency significantly and the accuracy of the results as well, see Fig. 2.

The robustness of the two-level approach can be demonstrated by casting all the Green's functions into closed forms with the use of the same approximation parameters, namely $T_{\circ 1}=400, N_{s_{1}}=50 T_{\circ 2}=5, N_{s_{2}}=100$. The normalized Green's functions of the vector and scalar potentials due to HED are obtained $\left(4 \pi G_{x x}^{A} / \mu_{o}\right.$, $4 \pi \epsilon_{3} G_{x}^{q}$ ) following the two-level approach (Apprx.) and evaluating the Sommerfeld integrals numerically (Exact), and given in Fig. 3. This test shows that the same set of approximation parameters can be used for any Green's function, that is, there is no need for an advance investigation of the Green's function and no need for any trial steps. The assessment of the robustness of the proposed approach also requires to study the sensitivity of the approximation parameters on the geometrical constants and the frequency. Therefore, the Green's functions for the vector and the scalar potentials are obtained in closed forms for the same geometrical constants and the same approximation parameters used above, but the frequency of operation is changed to $10 \mathrm{GHz}$, which is equivalent, in effect, to the change of the 
geometrical constants. It is observed that the agreements between the exact and approximate sets of data are still perfect and hence it is safe to conclude that the two-level approach proposed in this paper is very robust.

\section{References}

[1] Y. L. Chow, J. J. Yang, and D. F. Fang and G. E. Howard, "Closed form spatial Green's function for the thick substrate," IEEE Trans. Microwave Theory Tech., vol. MTT-39, pp. 588-592, Mar. 1991.

[2] M. I. Aksun and R. Mittra, "Derivation of closed-form Green's functions for a general microstrip geometries," IEEE Trans. Microwave Theory Tech., vol. MTT-40, pp. 20552062, Nov., 1992.

[3] G. Dural and M. I. Aksun, "Closed-form Green's functions for general sources and stratified media," in press.

[4] Y. Hua and T. K. Sarkar, "Generalized pencil-of-function method for extracting poles of an EM system from its transient response," IEEE Trans. Antennas Propagat., vol. AP-37, pp.229-234, Feb., 1989.

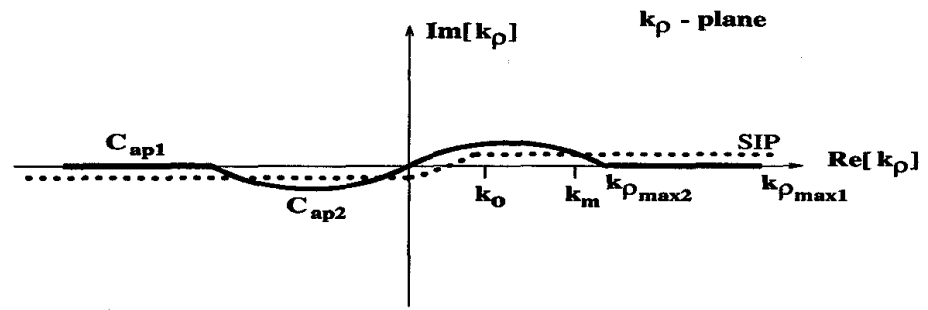

Figure 1: The paths $C_{a p 1}$ and $C_{a p 2}$ used in two-level approximation

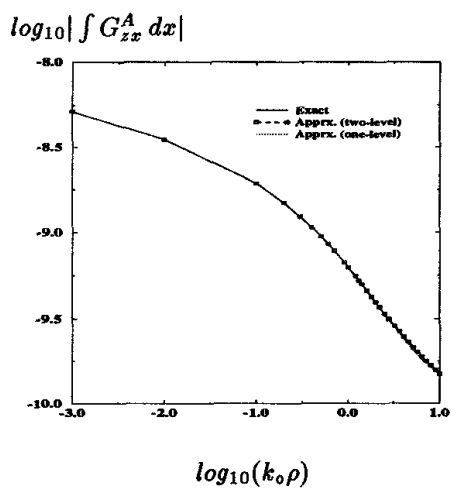

Figure 2: Magnitude of $\int G_{z x}^{A} d x$

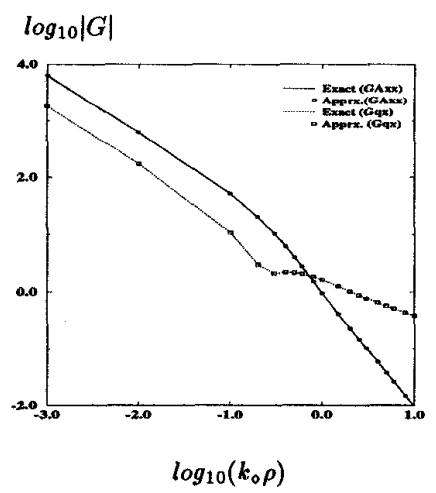

Figure 3: Normalized Magnitude of $G_{x x}^{A}$ and $G_{x}^{q e}$ 